UCRL--100891

DESO 002822

\title{
Prospects for a Soft X-Ray FEL Powered by a Relativistic-Klystron High-Gradient Accelerator (RK-HGA) by
}

H. D. Shay, W. A. Barletia, S. Yu, R. Schlueter, and G. A. Deis

I. INTRODUCTION

We present here the concept of $x$-ray FELs using high gain, single-pass amplifiers with electron beams accelerated in high gradient structures powered by relativistic kylstrons. Other authors ${ }^{1}$ have also considered $x$-ray FELs; the unique aspect of this paper is the use of high gradient acceleration. One of the authors (W.A.B.) has previously presented preliminary studies on this concept. 2 The intent in this paper is to display the results of a top level design sludy on a high gain FEL, to present its sensitivity to a variety of fabrication and tuning errors, to discuss several mechanisms for increasing gain yet more, and to present explicitly the output characteristics of such an FEL. The philosophy of the design study is to find a plausible operating point which employs existing or nearly existing state-of-the-art technologies while minimizing the accelerator and wiggler lengths. The notion is to distribute the technical risk as evenly as possible over the several technologies so that each must advance only slightly in order 10 make this design feasible. This study entailed no systematic investigation of possibie costs so that, for example, the sole criterion for balancing the trade-off between beam energy and wiggler length is that the two components have comparable lengths

In order to endow this study with some degree of tocus, we concentrated on satisfying the requirements for one of many possible applications, high resolution $x$-ray microscopy of biological structures in an aqueous environment. Specifically, the wavelength. power, and pulse width requirements stem clirectly from the objective of tomographic or holographic microscopy of resolution of several tens of nanometers over a field of view of one to ten microns. In order to have a sufficient number of photons scaltered from each resolution element to form an image, there must be a large fluence of photons through the sample, so large, in fact, tha! absorption can cause severe heating. London. el al ${ }^{3}$ have shown that the greatest ratio of signal to dose occurs at a wavelength of about $44 \AA$, at the carbon K-edge, and that, at this wavelength, scattering from a protein sphere of $15 \mathrm{~nm}$ diameter in a water environment has a cross section of about $10.14 \mathrm{~cm}^{2}$. For an adequate signal strength, about 1000 photons should be scattered per resolution elemient. These numbers imply that the incident $x$-ray 
fluence should be about $10^{18}$ photons $/ \mathrm{cm}^{2}$, about $45 \mathrm{~J} / \mathrm{cm}^{2}$, about $45 \mu \mathrm{J}$ over a field of view of $10 \times 10 \mu \mathrm{m}$. The energy absorption resulting from this fluence is so great that the image would suffer significant blurring if the exposure were any longer than about 30 ps. With the FEL architecture under consideration here the natural pulse length is 1-10ps, and so the required FEL peak power would be about 5-50MW.

One FEL architecture which can satisfy these requirements consists of a single pass, high gain wiggler amplifier with a highpeak-current electron beam accelerated in an RF high-gradient structure which is powered by a high-frequency relativistic klystron and which has a low-emittance injector. The FEL wiggler which amplifies exponentially from spontaneous emission would saturate as the signal power nears several tenths of a percent of the electron beam peak power and would not require tapering of the magnetic field near the end of the wiggler. This growth is illustrated by the GINGER [4] calculation shown in figure 1. An analytical estimate of the effective noise power is

$$
P_{A S E}=\frac{h(0)}{h} \frac{d P}{d \omega} \frac{d(0)}{\omega}
$$

where the power per unit frequency is

$$
\frac{d P}{d \omega}=\rho \frac{m c^{2} \gamma}{2 \pi}
$$

and $\rho$ is the Pierce parameter,

$$
\rho^{3}=-\frac{e}{m c^{2}} Z_{0} l \frac{\left.a_{w}^{2}[J]\right]^{2}}{16 \gamma^{3} A k_{w}^{2}} .
$$

For typical values ( peak current of $1 \mathrm{kA}$, beam energy of $1 \mathrm{GeV}$, beam radius of $65 \mu \mathrm{m}$, wiggler period of $2 \mathrm{~cm}$, a laser wavelength of $45 \mathrm{~A}$. and fractional bandwidth of $0.1 \%$ ), this estimate is $75 \mathrm{~W}$.

In this architecture, the wiggle motion is conlined to one plane, and the $x$-rays are consequently linearly polarized. The wiggler pole pieces are shaped [5] to provide coniinuous and equal focusing for the electron beam in both the horizontal and vertical planes: this technique has been successlully demonstrated on the 25m PALADIN wiggler [6]. For high gain, we consider rather short wiggle periods, $2.4 \mathrm{~cm}$; in the energy range of consideration (1. $2 \mathrm{GeV}$ ) and with the gap between opposing pole pieces greater than $\lambda_{w} / 4$ (great enough 10 permit high pumping speed and to avoid the 
excitation of wakefield or resistive wall instabilities [8]). the magnetic field required on axis is then great enough, $7-15 \mathrm{kG}$, to equal or to exceed the "Halbach limit" [7] for hybrid wigglers. Although the THUNDEF hybrid wiggler has been built with a wiggler period of $2 \mathrm{~cm}, a \mathrm{~g} / \lambda_{\mathrm{w}}$ of 0.25 , and a peak field on axis of 10kG [9], the range of fields under consideration here may imply the use of cryogenic, rare earth pole pieces [10] or superconducting windings. Since the current state-of-the-art in the random field errors of wiggler (in the PALADIN wiggler) is about $0.14 \%$, we consider a range of field errors somewhat smaller than $0.1 \%$. Similarly, since differential detectors can presently determine the centroid of a beam to better than $50 \mu \mathrm{m}$ in a beam pipe radius of $2 \mathrm{~cm}$ [11], we consider a range of steering better than $1 \%$ of the beam pipe radius.

The key features of the electron beam under consideration are its energy, $1.2 \mathrm{GeV}$, its normalized emittance, $1.100 \mathrm{~mm}-\mathrm{mrad}$, and its current, $0.3-10 \mathrm{kA}$. The electric break-down threshold in accelerating structures increases roughly as the square root of the frequency [12]. The use of $X$-band $(8-12 \mathrm{GHz}) \mathrm{RF}$ generated in relativistic klystrons permits a very high accelerating gradient [13], as much as $200 \mathrm{MeV} / \mathrm{m}$ in an unloaded structure driven at $11.4 \mathrm{GHz}$. Recent experiments, even without adequate repetition rate to provide any surface conditioning, have demonstrated the generation of $300 \mathrm{MW}$ of $11.4 \mathrm{GHz}$ of RF and its subsequent use in energizing a disk-loaded structure to an average, on-axis accelerating field of $100 \mathrm{MeV} / \mathrm{m}$ [14]. Sheffield and collaborators [15] have developed a photoinjector with a low emittance, ims of $10 \pi-40 \pi \mathrm{mm} \cdot \mathrm{mrad}$, a peak current of ${ }^{-} 00-400 \mathrm{~A}$, and a pulse length of 75ps. Compression of the pulse length from about 20-30ps to about 1-5 ps prior to acceleration to full energy can boost the peak current from several hurdied amperes to several kiloamperes. This compression would probably he conducted at some intermediate energy, like $50 \mathrm{MeV}$, to reduce the influence of space charge and to permit the preservation of the normalized emittance and to keep the induced energy spread small.

Most of this study has been conducted with numerical simulation of FEL amplifiers with the computer code FRED-3D [16]. which tracks the 3D betation motion of the electrons in wigglers with simulated field errors and compensatory steering. The radiation process is treated with the wiggle average KMR [17] equations. If tracks the evolution of several spatial modes of laser radialion. 


\section{DESIGN STUDY}

Before discussing the detailed numerical simulations, we first note some of the basic 1-D scaling parameters[1]. For typical values of current and energy and for a wiggler period of $2 \mathrm{~cm}$, the Pierce parameter is $O\left(10^{-3}\right)$. The e-folding gain length is thus

$$
L_{g}=\frac{\lambda_{w}}{4 \pi p}=1 \mathrm{~m}
$$

and the Rayleigh range is

$$
Z_{A} \equiv \frac{\pi r_{b}^{2}}{\lambda_{s}}-2 m \text {. }
$$

Since the gain length is shorter than the Rayleigh range, we anticipate that gain guiding is prevalent and 1D scaling does apply. The distance to saturation for ASE is LSAT $\approx O\left(\lambda_{w}\right)=O(10 \mathrm{~m})$ and the power at saturation is PSAT $=O\left(p P_{E B}\right)=O(11 / 2 \mathrm{GW})$. The limitation on the spread in the "parallel" energy is $\Delta \gamma\|/ \gamma\|<\rho / 4=410.4$. This estimate neglects the FEL lethargy.

As is apparent from these crude scaling relationships, we may anticipate higher gain for higher energy, higher current, and shorter wiggler period. Figure 2, plots of small signal gain at a wiggler length of $25 \mathrm{~m}$, illustrates the results of a series of FRED-3D simulations that support these notions. The conditions of the simulations are shown in the right-hand margin: a laser wavelength of $50 \mathrm{~A}$, three beam energies $(1,1.5$, and $2 \mathrm{GeV}$ ), two beam currents [ 3 and 10kA), a beam emittance (enclosing $90 \%$ of phase space) of 17.4 $\mathrm{mm}$-mrad, an energy spread of $10^{-3}$, rms wiggier errors of $10^{-3}$, and compensatory steering with a steering accuracy of $50 \mu \mathrm{m}$, and a steering interval of $4 \mathrm{~m}$. In these calculations, as in those throughout this paper, we have used a wavelength of $50 \mathrm{~A}$ rather than the more proper $44 \hat{A}$; this represents an inconsequential difference. In this. as in most of the simulations shown here, the small signa! gain represents the gain found at $25 \mathrm{~m}$ for a signal initially of $1 \mathrm{~W}$ power. a much lower power than actually anticipated for the noise source. Without tapering of $a_{w}$, the signal safurates at a power of of $O(10 G W)$ (depending on the energy and peak current). Because of lethargy, the exponential gain regime does not start for a few meters from the front of the wiggler, and so, for a small signal gain of $10^{10}$ or 23 e-folds, for example, there is about one e-folding every meter. In order to satisfy the microscopy requirement of about 10MW with an initial spontaneous noise power of $100 \mathrm{~W}$, about 
11 -foldings, in this example, would be necessary or about 11 meters of wiggler. The gain length decreases with decreasing wiggler period and, hence, increasing magnetic field, but wigglers become increasingly more difficult to build for smaller periods and higher fields. For example, the Halbach limit [7] for a SmCo hybrid wiggler with a ratio of pole gap to wiggler period of $1 / 4$ is about $10 \mathrm{kG}$, and is exceeded at $1.5 \mathrm{GeV}$ for all wiggler periods shorter than about $2.5 \mathrm{~cm}$. This limit can be increased by employing smaller gaps, but very small beam tubes poses several problems: low pumping speed, increased beam scraping, transverse wakefield inslabilities at pumping and diagnostic ports and the resistive wall instability. The last of these, resistive wall instability, probably does not present a very severe hazard oven in wiggler lengths as great as $25 \mathrm{~m}$. With the asymptotic formalism of Neil, etal. [8], we find that there are fewer than a few tenths of an e-fold of growth over the parameler range presented in figure 2. Still, in order to avoid excessively small gaps, some of the points on this figure might entail the use of superconducting coils or cryogenic poles.

Figure 3 shows that gain decreases with increasing normalized emittance. A larger emittance increases the "parallel" energy spread and decreases the current density. Shown in figure 3 are curves $f_{3}$ both $300 \mathrm{~A}$ and $1000 \mathrm{~A}$, which correspond roughly to having no temporal compression or a compression of a factor of about three in the electron bunches produced in the LANL gun [15]. Figure 4 displays the advantage of increased temporal compression and higher peak current, either for constant brightness or for constant emittance. Clearly careful compression preserving transverse emittance is important.

Tentatively then, designs in the area of $1-3 \mathrm{kA}, 1.5 \mathrm{GeV}, 20$ $\mathrm{mm}$-mrad, a wiggler length of about $10 \mathrm{~m}$, and a wiggler period of 2 $4 \mathrm{~cm}$ can satisfy the requirements for $x$-ray microscopy.

This study has examined the robustness of these designs to a variety of degrading factors: total energy spread, random wiggler field errors, jitter in the electron beam at the entrance to the wiggler, and steering accuracy. Figures 5, 6, and 7 show these sensitivities. While the nominal design point of an energy spread of $10^{\circ} 3$, a steering accuracy of about $50 \mu \mathrm{m}$, and an rms random wiggler field errors of about $10 \cdot 3$, are adequate to provide the needed gain, improvement of $50 \%$ in any of the parameters could substantially increase the gain. Then 10o, simulations have also shown that the higher peak currents also reduce the sensitivity of the gain to these factors. 


\section{ADDITIONAL TECHNIQUES FOR IMPROVING GAIN}

While the designs shown above seem adequate for providing the gain for $x$-ray micrescopy, we have investigated several additional techniques for achieving yet higher gain as a hedge against unforeseen difiiculties.

As alluded to above, properly curved pole tips can focus the electron beam equally in vertical and horizontal directions. If additional focusing is added, either by the introduction of external quadrupole magnets or by a weakly ionized channel [18], then the current density and, hence, the Pierce parameter are increased. Figure 8 illustrates the resulting improvernent in the gain, about two orders of magnitude, when the electron beam radius is decreased by a factor of about two from that attained with the "natural" focusing by the fields of the curved pole tips. One possible disadvantage to this technique of tighter focusing is that the sensitivity to field errors and steering accuracy is conccmittantly increased.

It is well known that higher harmonics are emitted along with the FEL fundamental [19]. In particular, odd order harmonics in a linear wiggler are generated simply because an electron trajectory does not have a constant radius of curvature. Of course, tapering is not possible for the higher harmonics because the bunching is still at the fundamental frequency. In the exponential gain regime however, one technique might be to tune for resonance at a wavelength three times longer (150A) and then to use its third harmonic. Figure 9 shows a series of calculation conducted with a code NUTMEG [20], a code similar to the $2 D$ version of FRED, but which follows a higher harmonic along with the fundamental. The third harmonic $(50 A)$ uniformly has lower gain than the fundamental $(150 \mathrm{~A})$, but, for the longer wiggler periods, has higher gain than that achieved in a wiggler tuned for a fundamental at $50 \mathrm{~A}$. This technique can permit the fabrication of much less challenging wigglers with periods of $4.5 \mathrm{~cm}$, rather than with $2.3 \mathrm{~cm}$.

\section{FEL BEAM CHARACTERISTICS}

Table 1 summarizes the output characteristics of an $x$-ray FEL.

As evident in figure 1, the bandwidth initially decreases as $1 / 2 N_{\rho}$ : simulations indicated that, for a long enough wiggler, the lower bound on $\Delta \lambda / \lambda$ is determined by the energy spread in the 
electron beam so that bandwidth is never smaller than $2 \Delta \gamma / \gamma$. With bandwidths of about $0.2 \%$, the longitudinal coherence length $\left(\lambda^{2} / \Delta \lambda\right)$ is $2.5 \mu \mathrm{m}$, just right for cellular microscopy.

Despite the fact that the spontaneous noise has a random distribution of spatial modes, the output has predominantly the TEM 00 mode. Because of strong gain guiding, after about 10 meters, over $90 \%$ of the power is carried in the TEM0O Gaussian mode as shown by the FRED-3D calculation illustrated in figure 10 .

Depending on the length of the wiggler, 10 to 25 meters, it is possible to attain peak powers of $10^{8}$ to $10^{9} \mathrm{~W}$. For a pulse length of $1-10 p s$, each $x$-ray pulse would have 0.1 to $1.0 \mathrm{~mJ}$ or $2.5 \times 10^{12}$ to $2.5 \times 10^{13}$ per pulse. Since the beam radius is about $100 \mu \mathrm{m}$ and its far field divergence is about $20 \mu \mathrm{rad}$ (half angle), each pulse would have a spectral brilliance of $2 \times 10^{29} 102 \times 10^{30}$ photons $/\left(\mathrm{sec} \mathrm{mm}^{2}\right.$ $\left.\mathrm{mrad}^{2} 0.1 \% \mathrm{BW}\right)$, which is much higher than is possible from storage ring sources.

The pulse power network and LIA driving this FEL would be similar to existing devices which can in some case operate $\mathrm{CW}$ at tens of Hertz. We anticipate then that repetition rates of $1-10 \mathrm{~Hz}$ would be quite feasible.

\section{SUMMARY}

We have identified one of many possible applications for an $x$-ray FEL of high spectral brilliance: $x$-ray microscopic holography or tomography. Its light source requirements are a wavelength of $44 A$, a pulse length of 10ps or less, and an energy per pulse of about $50 \mu \mathrm{J}$. We have calculated the performance of an FEL design which cepitalizes upon the recent advances in relativistic klysirons, $x$ band high gradient accelerators, photoinjectors, and high precision wigglers. Such an FEL would be a sirigle pass amplifier with exponential growth from spontaneous noise. It would have 10.15 meters of wiggler with a period of $2.4 \mathrm{~cm}$, rms random field errors of $0.1 \%$ and steering accuracy of $50 \mu \mathrm{m}$. It would be driven by an electron beam of about $1.5 \mathrm{GeV}$ (accelerated in a high gradient structure about $7.5 \mathrm{~m}$ long), peak current of $1-3 \mathrm{kA}$, an energy spread of $0.1 \%$, and an emittance of about $20 \mathrm{~mm}$-mrad. These design parameters have been estimated with due consideration to possible variation in errors in tuning and labrication. All of the relevant technologies are rapidly approaching the capability of attaining these parameters. We have alsc identified several techniques for producing additional gain. Our FRED-3D and NUTMEG simuiations 
suggest that the output characteristics of such an $x$-ray FEL would be ideal for cellular $x$-ray microscopy.

Work performed jointly under the auspices of the U.S. Department of Energy by Lawrence Livermore National Laboratory under contract W-7405-ENG-48. 


\section{FOOTNOTES}

[1] K.J. Kim, Phys. Rev. Lett. 57 (1986), 1871; J.C. Goldstein, B.D. McVey, and J.C. Elliot, Nuclear Instruments and Methods in Physics Research A272 (1988), 177; C. Pelegrini, Nuclear Instruments and Methods in Physics Research A239 (1985), 127.

[2] W. A. Barletta, M. Atac, A. Bhomik, B. Bobbs, D.B. Cline, R.A. Cover, F.P. Dixon, J. Gallardo, J. Kolonko, C. Pellegrini, G. Rakowsky, X. Wang, "A Compact X-Ray Free Electron Laser," Falmouth Conference on $X$-Ray Lasers, September 9, 1938.

[3] R.A. London, M.D. Rosen, and J.E. Trebes, to appear in Applied Optics 28 (1989).

[4j W.A. Fawley, private communication.

[5] E.T. Scharlemann, "Wiggle Plane Focusing in Linear Wigglers," LLNL Report UCFL-92429 (1985).

[6] G.A. Deis, A.R. Harvey, C.D. Parkinson, D. Prosnitz, J. Rego, and E. T. Scharlemann, IEEE Trans. on Magnetics 24 (1988), 1090.

[7] K. Halbach, J. de Phys. 44 (1983), Cl-211.

[8] G.J. Caporaso, W.A. Barletta, and V.K. Neil, Particle Accelerators 11 (1980), 71.

[9] K.E. Robinson, D.C. Quimby, J.M. Slater, T.L. Churchill, A. Pindroh, and A. Valla, Nuclear Instruments and Methods in Physics Research A250 (1986), 100.

[10] R. Hoard, et_al, "Field Enhancement of 12.5-T Magnet using Holmium Poles," UCRL-90638 (1984).

[11] B. Kulke, H.D. Shay, $\vec{F}$. Coffield, and R. Holmes, Nuclear Instruments and Methods in Physics Research A272 (1987), 241.

[12] G.A. Lowe and J.W. Wang. "RF Breakdown Studies in Room Temperature Electron Linac Structures," SLAC-PUB-4647 (1988).

[13] A.M. Sessler and S.S. Yu, "Relativistic Klystron Two-Beam Accelerator," Phys. Rev. Lett. 58 (1987), 2439.

[14] M.A. Allen, F.S. Callin, H. Deruyter, K.R. Eppley, K.S.Fant, W.R. Fowkes, H.A. Hoag, R.F. Koontz, T.L. Lavine, G.A. Lowe, R.H. Miller, R.D. Fiuth, A.E. Vlieks, J.W. Wang, D.P. Aalberts, J.K. Boyd, T. Houck, R. D. Ryne, G. A. Westenskow, S. S. Yu, D.B. Hopkins, A.M. Sessler, J. Haimson, B. Mecklenburg. "Recent Progress in Relativistic Klystron Research," XIV International Conference on High Erergy Accelerators, Tsukuba, Japan, August 22-26, 1989. 
[15] R.L. Sheffield, E.R. Gray, and J.S. Fraser, Nuclear Instruments and Methods in Physics Research A272 (1988), 222.

[16] E.T. Scharlemann and W.M. Fawley, SPIE Symp. c.n Optics and Optoelectronics Systems (1986), Lawrence Livermore National Laboratory, Report UCRL-94258.

[17] N.M Krall, P.L. Morton, and M.N. Rosenbluth, "Free Electron Lasers with Variable Parameter Wigglers," IEEE J. Quant. Elec. QE-17 (1981), 1436.

[18] W. A. Barletta and A. M. Sessler, "Radiation from Fine, Intense, Self-Focused Bams at High Eriergy," Proceedings of INFN International School on Electromagnetic Rasiation and Particle Beam Acceleration, Varenna, Italy (June, 1988).

[19] W.B. Colson, "The Nonlinear Wave Equation for Higher Harmonic's in Free Electron Lasers," IEEE J. Quant. Elec. QE-17 (1981), 1417. [20] E.T. Scharlemann, private communication. 


\section{FIGURE CAPTIONS}

Figure 1.

This graph shows the laser power as a function of the axial distance along the wiggler as calculated in the computer code GINGER. The electron beam and wiggler parameters appear on the right hand margin. The spectral distribution of the $x$-rays between 48 and $52 A$ are depicted at the start of the wiggler and at 4 and 8 meters.

Figure 2.

This plot illustrates several series of FRED-3D predictions foi small signal gain as a function of wiggler period for several beam energies and currents. The calculational parameters are noted on the right hand margin. In all cases the electron beam emittance (that which encompasses $90 \%$ of the phase space) was $17.4 \mathrm{~mm}$-mrad. The initial $x$-ray power was $1 \mathrm{~W}$, considerably lower than the anticipated $100 \mathrm{~W}$ of noise power at 50A. Saturation is observed above 10GW. Figure 3.

This sraph shows the small signal gain as a function of the normalized emittance for two peak currents.

Figure 4.

This graph shows the calculated variation of gain with peak current either for constant brightness or normalized emittance. Figure 5.

This plot depicts the variation of gain with the energy spread in the electron beam.

Figure 6 .

This plot depicts the variation of gain with the rms average random wiggler field error. In the FRED-3D calculations, the same random seed was used in generating the pseudo-rarciom distribution of field errors.

Figure 7

This plot shows the calculaied gain as a functisin of the displacement of the electron beam from the wiggler axis. The wiggler magnetic field and steering was tuned in the calculation without any beam displacement and held constant for the other two calculations to represent a situation of rapid and unexpected beam flutter. 


\section{Figure8}

This graph shows the consequence of adding additional focusing to the wiggler.

Figure 9.

This graph shows gain as calculated by the multiple harmonic code NUTMEG for the fundamental at $50 \mathrm{~A}$ and $150 \mathrm{~A}$ and for the third harmonic at $50 \AA$ when the viggler is tuned to $150 \AA$.

Figure 10.

This graph shows the power in each of the azimuthal modes as a function of distance along the wiggler. The inilial conditions were a random mixture of Gauss-Laguerre modes for azimuthal $m$ values of 0 and \pm 1 and a range of radial $n$ values; the modes were permitted to evolve into modes with $m$ values of $0, \pm 1$, and \pm 2 . Because of gain guiding, the TEM 00 Gaussian mode naturally predominated.

Table 1.

This table summarizes the predicted output characteristics of an $x$-ray FEL of the nominal design described here. 


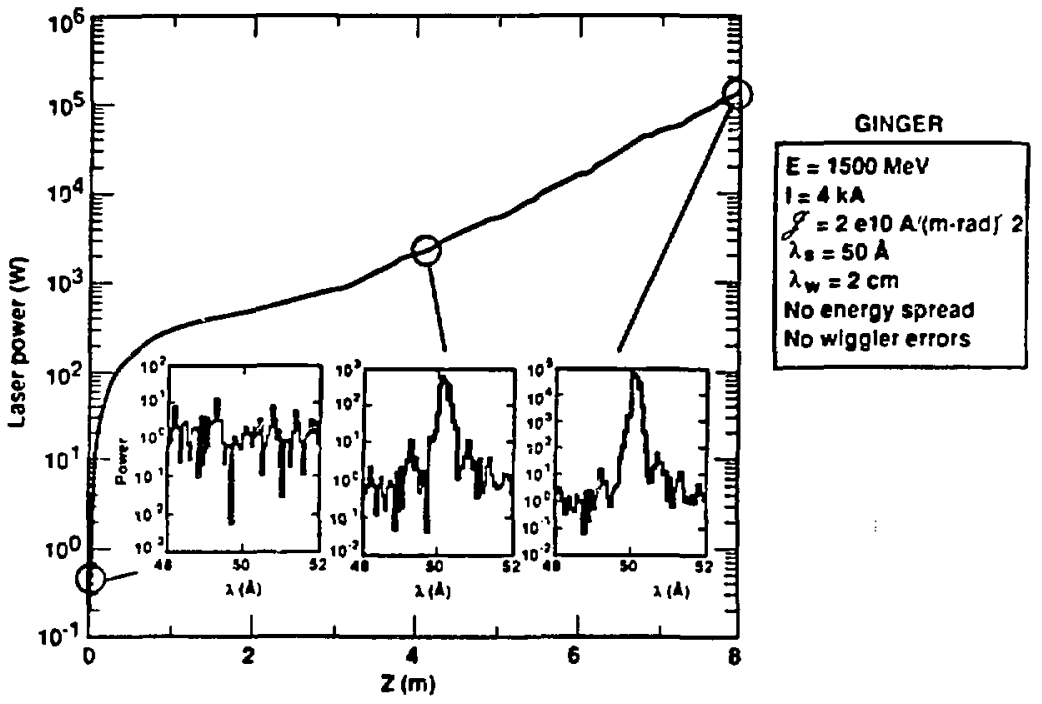

Fig. I 


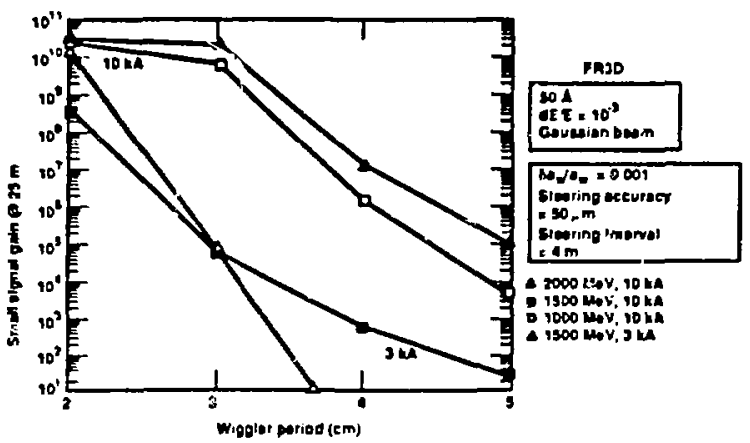

FIg. 2 


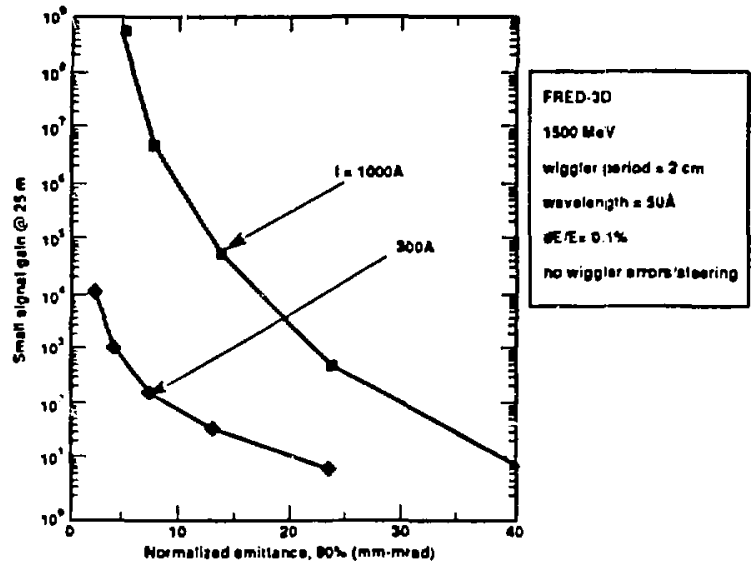

FIg. 3 


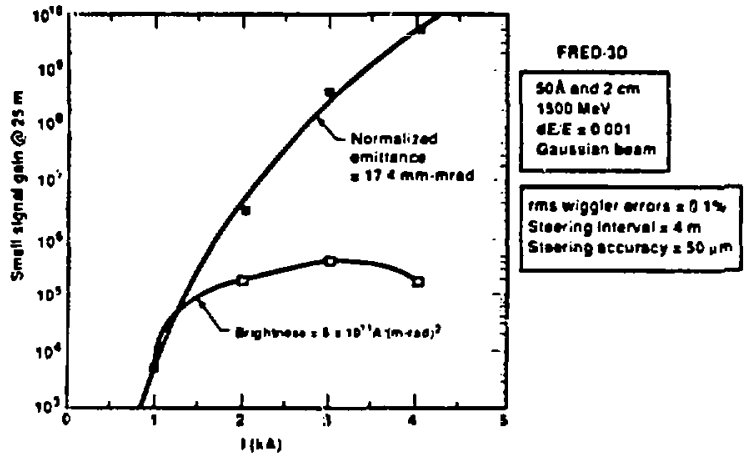

Fis. 4 


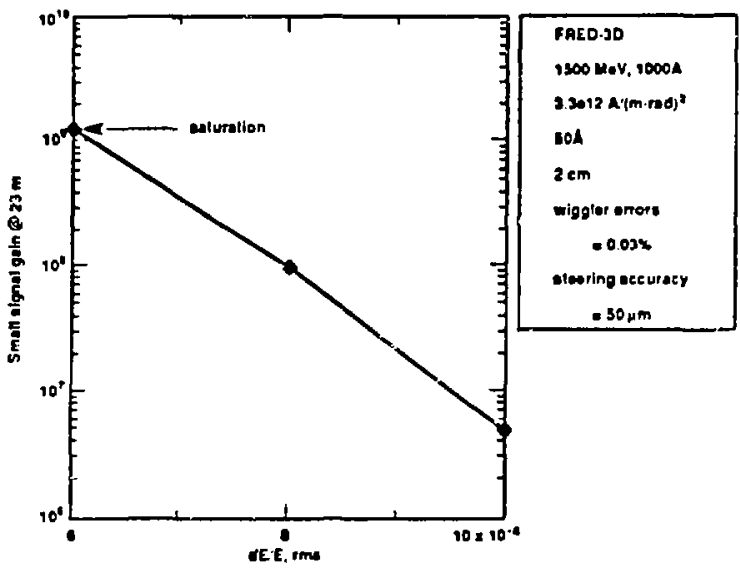

Fig. S 


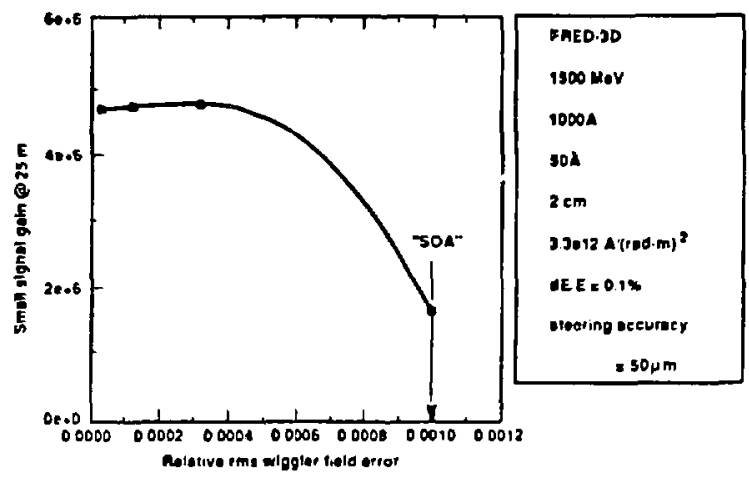

FIg. 6 


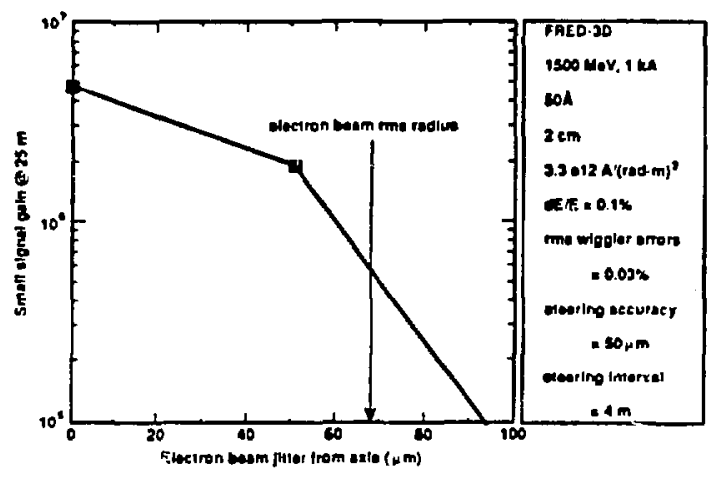

F1. 7 


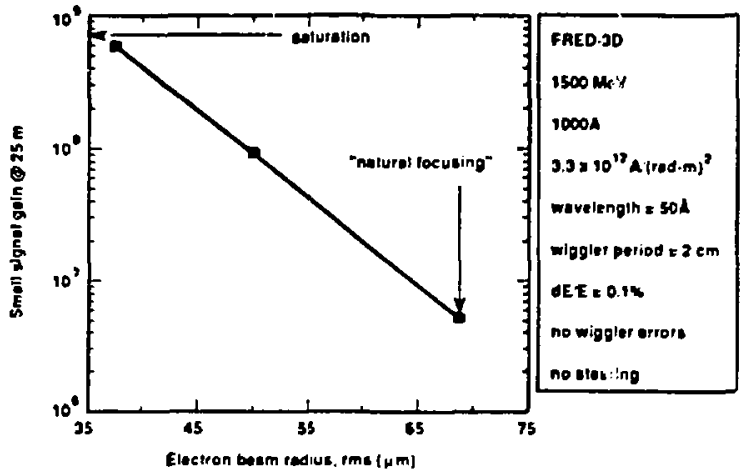

F18. 8 


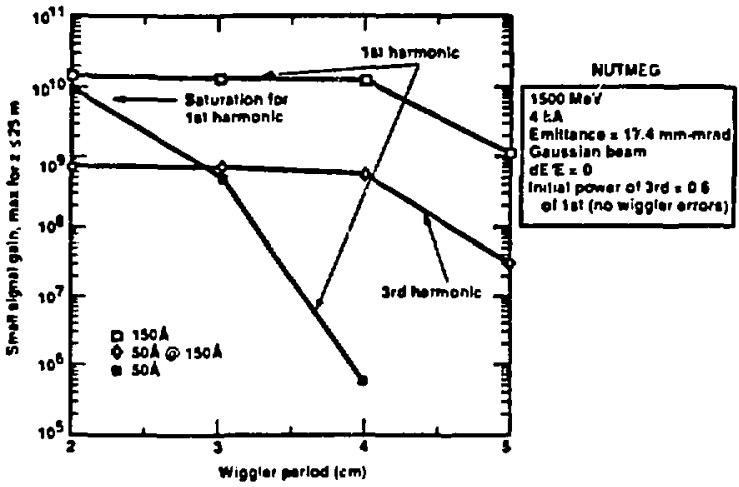

F18. 9 


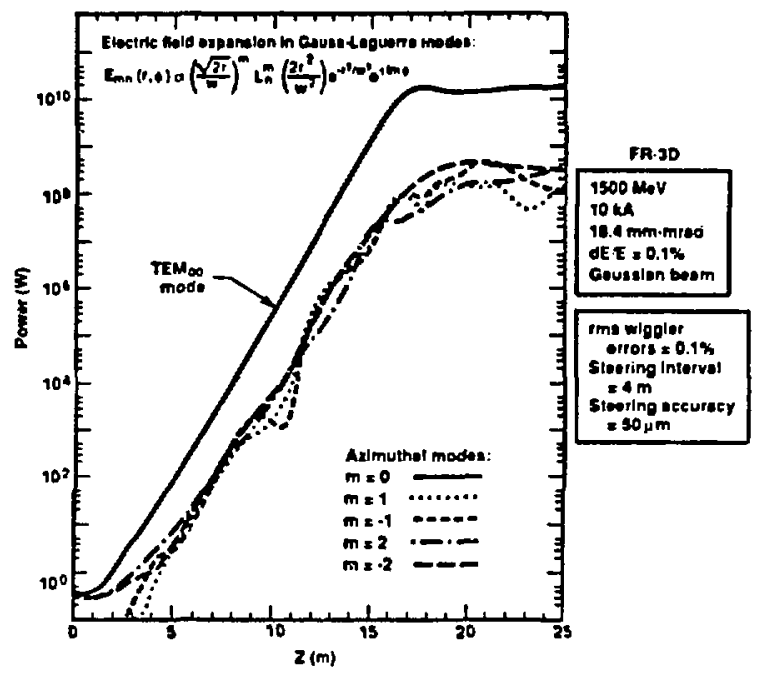

Fig. 10 


\section{IABLEA}

Characteristic

$\boldsymbol{\lambda}$

$\Delta \lambda \lambda$

Instantaneous power

Beam radius at exit of FEL

Beam divergence at exit of FEL

Spectral brilliance

Pulse length

Ene:gy/pulse

Transverse Coherence

Longitudinal Coherence, $\frac{\lambda^{2}}{\Delta \lambda}$

Repetition rate
Value

$50 \mathrm{~A}$

$0.2 \%$

$=10^{8} \cdot 10^{9} \mathrm{~W}$

$=100 \mu \mathrm{m}$

- $20 \mu \mathrm{rad}$

$=2 \times 10^{29} .2 \times 10^{30}$ photons (sec $\cdot \mathrm{mm}^{2} \cdot \mathrm{mrad}^{2} 0.1 \% \mathrm{BW}$ )

$.1 \cdot 10 \mathrm{ps}$

* $0.1-1 \mathrm{~mJ}$

- $85 \%$ in TEM $_{\infty}$ mode

$=2.5 \mu \mathrm{m}$

$1 \cdot 10 \mathrm{~Hz}$ 